Foro Interno. Anuario de Teoría Política

ISSN: 1578-4576

http://dx.doi.org/10.5209/FOIN.61510

\title{
La globalización en la redefinición de lo político
}

\author{
Daniel Flores Gaucin ${ }^{1}$
}

Recibido: 12 de junio de 2017 / Aceptado: 8 de marzo de 2018

Resumen. El constante aumento de la intensidad con la que se globalizan los diferentes ámbitos de la vida en la mayoría de las sociedades actuales ha intensificado, a su vez, algunos de los desafíos que los principales actores a nivel global habían estado enfrentando las últimas décadas. Poner de relieve estos desafíos, ahora globales, permite resaltar una serie de complicaciones de talante también global, a las que, no obstante, habrán de hacer frente Estados cuyo poder se encuentra constreñido territorialmente. Ante esta dialéctica que señala de manera particular la gravedad y urgencia de los riesgos globales, los Estados se ven obligados a enfrentar, además, un cuestionamiento de carácter esencial. ¿Es pertinente una estructura de poder que, por sí misma, no es capaz de garantizar la seguridad de quienes la componen? ¿Qué modelo político o qué política global podría, en el corto plazo, solventar las complicaciones y sobreponerse a los desafíos de esta modernidad globalizada?

Palabras clave: Globalización; Estado; riesgo global; cooperación internacional; modernidad.

\section{[en] Globalization and the Redefinition of Politics}

Abstract. The continuous increase in the intensity with which varying spheres of life are now globalized in most countries has also intensified some of the challenges that political actors at a global level have been facing in recent decades. Complications that actors face at a global level will nevertheless need to be addressed by territorially constrained States. Considering this dialectic reality that points out the gravity and urgency of global risks, States are also forced to face a critical issue: Is a power structure relevant if it is unable to guarantee by itself the safety of those who compose it? What political system, or global policy, could in the short term overcome the complications and challenges of this globalized modernity?

Keywords: Globalization; State; global risk; international cooperation; modernity.

Cómo citar: Daniel Flores Gaucin, "La globalización en la redefinición de lo político": Foro Interno. Anuario de Teoría Política, vol. 18 (2018), pp. 51-73.

\footnotetext{
Universidad Autónoma de Madrid (España)

E-mail: flores.gaucin@gmail.com
} 


\section{La política frente a los desafíos globales}

\subsection{Menos de tres minutos para el fin del mundo}

El reloj del fin del mundo marca las 23:57:30. Para los miembros del comité de ciencia y seguridad del Bulletin of the Atomic Scientists, el fin de la humanidad es una amenaza latente. El Bulletin, fundado en 1945 por los científicos del Proyecto Manhattan que "no podían mantenerse al margen de las consecuencias de su trabajo" y que desde entonces evalúa los avances científicos de acuerdo con su potencial beneficio o daño al mundo y sus habitantes, considera que los principales elementos de riesgo en la actualidad son el cambio climático, el arsenal nuclear y el desarrollo de nuevas tecnologías que, utilizadas de manera negligente o malintencionada, pudieran representar un riesgo para la vida en este planeta. El reloj solo había estado más cerca de la medianoche en 1953, luego de que tanto Estados Unidos como la Unión Soviética respectivamente realizaran, a manera de prueba, la detonación de una bomba de hidrógeno. Los años siguientes la humanidad pudo contemplar cómo las manecillas se alejaban de la hora funesta a partir de que, desde 1991, se llevara a cabo la firma de tres tratados de desarme nuclear entre las dos potencias militares de la Guerra Fría. En los últimos años, sin embargo, la principal fuente del peligro ha pasado a fundamentarse sobre todo en el cambio climático. Los científicos encargados de ajustar la hora del doomsday clock confirman que la situación actual todavía no adopta un carácter irreversible, pero señalan un serio fracaso por parte de los dirigentes a la hora tomar las medidas necesarias para "alcanzar lo que representa su más importante responsabilidad: asegurar y preservar el bienestar de la civilización humana" 2 . Urgen a los gobiernos para que tomen acciones de manera inmediata e imploran a los ciudadanos del mundo a que, a su vez, demanden de sus dirigentes una estrategia que pueda contrarrestar la amenaza que se ciñe sobre ellos ${ }^{34}$.

No obstante que el riesgo del estallido de un conflicto nuclear durante la Guerra Fría hubiese representado una alarma mayor que la se vive actualmente, lo cierto es que el problema que enfrenta la humanidad ahora amerita una solución cuya realización parece mucho más complicada que entonces. La razón principal es que esta vez no se reduce a una conciliación entre dos potencias económico-militares, sino que tanto problemática como solución tienen un carácter global. En lo que se refiere

2 John Mecklin (ed.), "It is Still 3 Minutes to Midnight, 2016 Doomsday Clock Statement": Bulletin of the Atomic Scientists (2016). Disponible en: https://goo.gl/fTVS64 (7-6-2017).

3 Si bien los riesgos para la humanidad pueden ser catalogados entre antropogénicos y no antropogénicos, los del primer orden resultan mucho más preocupantes debido a que la probabilidad de que deriven en una catástrofe es considerablemente mayor. Acerca de esta categorización de los riesgos véase Nick Beckstead y Toby Ord, "Managing Existential Eisk from Emerging Technologies", en Mark Peplow (ed.), Annual Report of the Government Chief Scientific Advisor 2014. Innovation: Managing Risk, Not Avoiding It, Government Office for Science, London, 2014, pp. 115-120; y Cotton-Barratt et al., Global Catastrophic Risks 2016, The Global Challenges Foundation, Global Priorities Proyect. Disponible en: https://goo.gl/8w5ZYs (6-7-2017). Si tomamos en cuenta que, como especie, no contamos aún con las herramientas necesarias para hacer frente o siquiera prever con suficiente margen de tiempo las catástrofes de orden natural ante las que somos susceptibles, resulta claro que el tipo de riesgos que nos interesa abordar aquí son los que pudieran tener una solución que esté en manos humanas.

4 John Mecklin (ed.), "It is Two and a Half Minutes to Midnight, 2017 Doomsday Clock Statement”: Bulletin of the Atomic Scientists (2017). Disponible en: https://goo.gl/o3XFkz (28-9-2017). 
al armamento nuclear: Estados Unidos y Rusia, a pesar de que juntos poseen cerca del $93 \%$ del total de armas nucleares en el mundo, no solo han estancado cualquier pretensión de desarme, sino que ahora sus líderes esgrimen sendas retóricas belicistas que amenazan con reavivar la perniciosa carrera armamentística de antaño. Mientras tanto, el resto de países que cuentan con este tipo de arsenal en su poder se encuentran, o bien modernizándolo o bien desarrollándolo. De la lista que incluye al Reino Unido, Francia, China, Israel, India y Pakistán, destaca en lo particular Corea del Norte, país que ha confirmado su pertenencia a esta lista de forma definitiva tras la exitosa prueba nuclear efectuada el 3 de septiembre de $2017^{5}$. Si realmente se desea llegar a un acuerdo en pos de mejorar esta situación, este tendría que contar, al menos, con la firme voluntad de cada uno de estos países, pues se estima poco probable que una nación decida iniciar un programa de desarme nuclear, a la vez que ve cómo aumenta el arsenal de alguna otra, con la que pudiera estar en conflicto o a la que considere un enemigo potencial. Si más países se ven inmiscuidos en esta lógica belicista de buscar constantemente equiparar o superar el arsenal del país vecino o enemigo, cuyo ejemplo patente podemos encontrar en la relación India-Pakistán ${ }^{6}$, es lógico pensar en la posibilidad de que se desate una reacción en cadena que incremente enormemente los riesgos de un desastre nuclear.

Sin embargo, los problemas relacionados con el desarrollo tecnológico van allá del ámbito armamentístico. El propio Stephen Hawking, una de las voces más aclamadas entre los entusiastas por el desarrollo tecnológico (o precisamente debido a ello), durante su vida nunca dejó de alertar acerca de sus peligros:

La mayoría de las amenazas que enfrentamos son consecuencia de los avances que hemos hecho en ciencia y tecnología. Como no vamos a detener el progreso o a revertirlo, debemos entonces identificar los riesgos y ponerlos bajo control. Soy optimista y creo que podemos hacerlo ${ }^{7}$.

Destacan los relacionados con la ingeniería genética y la inteligencia artificial. En ambos casos la mayor causa de riesgo tiene que ver con un uso negligente de esta tecnología o con que su avance sea tal, que el acceso a estas resulte demasiado sencillo para organizaciones criminales o grupos terroristas.

Las mejores formas de mitigar estos riesgos pasan por una adecuada regulación en la investigación y desarrollo de estas tecnologías, así como de un estricto control sobre sus resultados. Sin embargo, la cooperación internacional nuevamente resulta ser un elemento esencial a la hora de alcanzar este cometido. Hoy en día, por ejemplo, es completamente posible para un hacker ubicado en Nueva Zelanda robar información clasificada de carácter militar o financiera de una computadora ubicada en Estados

\footnotetext{
Por si fuera poco, esta prueba ha estado acompañada de una serie de declaraciones desafiantes dirigidas contra los Estados Unidos que han sido respondidas con el mismo tenor por parte del presidente Donald Trump a través de Twitter. Todo ello ha alimentado la zozobra ante una posible agresión nuclear. Prueba de ello es que Kevin Rudd, ex primer ministro de Australia y ahora presidente de un renombrado think tank, ha declarado recientemente (septiembre de 2017) que estimaba la probabilidad de una agresión nuclear como consecuencia de esta alza en las tensiones en uno entre cuatro.

6 Michael Kugelman, "Kashmir Conflict: Do Nuclear Weapons Prevent All-out War?": CNN (30-9-2016). Disponible en: https://goo.gl/jZU7NG (6-7-2017).

7 David Shukman, "Hawking: Humans at Risk of Lethal 'Own Goal”": BBC (19-1-2016). Disponible en: https:// goo.gl/XfXt5J (6-7-2017).
} 
Unidos para después almacenarla en un servidor clandestino de Afganistán; con la posibilidad, por supuesto, de filtrar dicha información a través de internet, logrando así que en cuestión de minutos miles de personas alrededor del mundo puedan disponer de estos datos para el propósito que sea. Todo ello, con la confianza de que la posibilidad de ser rastreado o llevado ante las autoridades afectadas es considerablemente baja. La receta del desastre sería que sea un grupo terrorista y no un hacker, desde un lugar indeterminado del planeta con una insuficiente regulación de seguridad, sea quien, haciendo uso en este caso de la biotecnología, desarrollase un virus epidemiológico altamente letal y contagioso con el objetivo de desatar sus efectos en otro lugar del mundo, donde los frutos de la devastación se alineen mejor a cualesquiera que sean los objetivos de su agenda ${ }^{8}$. El hecho de que las agencias estadounidenses de inteligencia hayan sido incapaces de prevenir la interferencia del gobierno ruso en las elecciones de 2016 es una situación que devela la manifiesta vulnerabilidad de los Estados ante un posible uso malintencionado de las tecnologías de la información.

Por último, el que ahora es el mayor de los problemas: el deterioro del medio ambiente. Es probablemente el de más difícil solución sobre todo porque, en este caso, no es posible ubicar a un individuo o grupo en particular como los causantes del cambio climático; no hay un enemigo postrado frente a nosotros al cual combatir, al cual temer, al cual culpar. En este caso, el enemigo se encuentra en cierta medida dentro de cada uno de nosotros; son todos los países, cada uno con algún grado de implicación, los que han contribuido y contribuyen día con día a hacer de este problema el más crítico para la humanidad. La emisión de gases de efecto invernadero es una circunstancia que intrínsecamente forma parte del desarrollo y del funcionamiento de la civilización pues es ocasionada principalmente por los procesos de generación de energía, por la industria y por el transporte ${ }^{9}$. Sin embargo, si a nivel global se implementaran medidas de eficiencia energética y se incrementaran los esfuerzos por incentivar el desarrollo y la utilización de alternativas funcionales a los combustibles fósiles como las llamadas energías renovables, sería posible limitar de forma importante la cantidad de emisiones. No obstante, y a pesar del riesgo, lo que parece imposibilitar en mayor medida la toma de acciones contundentes en este sentido no es solo la relevancia que aún tiene en la economía global la utilización de estos combustibles, sino también el valor que representan como recursos en sí mismos dentro de la escena geopolítica.

\subsection{El calentamiento global como riesgo esencial}

La cantidad precisa de gigatoneladas de $\mathrm{CO}_{2}$ y la dimensión exacta de la catástrofe son datos que permanecen imposibles de determinar por ahora ya que dependen de cuán susceptible pueda ser el medio ambiente a la cantidad total de dióxido de carbono en él. En este sentido se estima que, en un escenario moderado, si se mantiene el aumento en las emisiones a nivel mundial (2.2\% anual entre 2000 y 2010$)$, las consecuencias serían que para finales del siglo ventiuno la temperatura media global habría aumentado entre $3.1{ }^{\circ} \mathrm{C}$ y $5.2{ }^{\circ} \mathrm{C}$ por encima de los niveles preindus-

Cotton-Barratt et al., Global Catastrophic Risks 2016.

$9 \quad$ IPCC, Climate Change 2014: Mitigation of Climate Change. Contribution of Working Group III to the Fifth Assessment Report of the Intergovernmental Panel on Climate Change, Cambridge University Press, Cambridge, 2014, pp. 41-50. Disponible en: https://goo.gl/ZKho2K (6-7-2017). 
triales (actualmente se encuentra alrededor de $0.8^{\circ} \mathrm{C}$ por encima). Se sabe que con el aumento en la temperatura promedio también se incrementan el nivel del mar, el promedio de precipitaciones a nivel global y la acidificación de los océanos ${ }^{10}$. Es por ello por lo que los expertos anticipan que niveles de entre $1{ }^{\circ} \mathrm{C}$ y $2{ }^{\circ} \mathrm{C}$ conllevarían un alto riesgo a experimentar daños irreversibles al ecosistema y fenómenos meteorológicos extremos. Mientras que un aumento a niveles cercanos a $4{ }^{\circ} \mathrm{C}$ tendría como consecuencia "importantes extinciones de especies, inseguridad alimentaria mundial y regional, limitaciones para actividades humanas normales y capacidad potencial limitada para la adaptación en algunos casos" ${ }^{\prime 1}$. Si además consideramos que en un escenario pesimista la temperatura media global podría llegar a aumentar hasta en $7.8^{\circ} \mathrm{C}$, resulta más clara la dimensión de una posible catástrofe.

A partir de la Conferencia de Cancún (2010) se ha establecido cierto consenso en fijar los $2{ }^{\circ} \mathrm{C}$ como la cifra límite en el aumento medio de la temperatura y es necesario decir que algo se ha hecho a nivel internacional por lograr este cometido. El más importante avance realizado en este sentido fue concretado en diciembre de 2015, cuando 194 países firmaron el acuerdo de París en el que cada nación dejó estipuladas diversas "promesas" encaminadas a reducir la utilización de combustibles fósiles e implementar políticas de estímulo a favor del desarrollo de energías renovables, todo ello con el objetivo de mantener el nivel de aumento en la temperatura media global "muy por debajo" del límite de $2{ }^{\circ} \mathrm{C}$.

Tristemente, hay muy pocos argumentos sobre los cuales soportar una actitud optimista con relación a París. Hoy el acuerdo enfrenta principalmente tres grandes retos que debe superar para alcanzar su cometido:

(1) Garantizar la eficacia en el cumplimiento de las disposiciones. Hay muchas voces críticas que apuntan sobre todo hacia el hecho de que las promesas firmadas en el acuerdo no sean obligatorias ni incorporen medidas de sanción, sino que se encuentran fundamentadas en mera buena voluntad, dando al acuerdo un enfoque "suave" que simplemente no funcionará ${ }^{12}$. Señalan, además, cómo en realidad estas promesas no son el resultado de una verdadera negociación sino de las estimaciones propias de los países firmantes de acuerdo con un escenario de business as usual ${ }^{13}$.

10 John Reilly et al., Energy \& Climate Outlook. Perspectives From 2015, Massachusetts Institute of Technology, Cambridge, 2015. Disponible en: https://goo.gl/6JREiQ (6-7-2017).

11 IPCC, Climate Change 2014: Impacts, Adaptation, and Vulnerability. Part A: Global and Sectoral Part A: Global and Sectoral Aspects. Contribution of Working Group II to the Fifth Assessment Report of the Intergovernmental Panel on Climate Change, Cambridge University Press, Cambridge, 2014, p. 63. Disponible en: https://goo.gl/xdWxzP (6-7-2018).

12 Para consultar algunas perspectivas críticas sobre el tema de los acuerdos climáticos no vinculantes, véase: Joseph Stiglitz, "Overcoming the Copenhagen Failure with Flexible Commitments": Economics of Energy and Environmental Policy, vol. 4, n. ${ }^{\circ} 2$ (2015), pp. 29-36; Felix R. FitzRoy y Elissaios Papyrakis, An Introduction to Climate Change Economics and Policy, Routledge, London, 2016; y Sergey Paltsev, "The Complicated Geopolitics of Renewable Energy": Bulletin of the Atomic Scientists, vol. 72, n. ${ }^{\circ} 6$ (2016), pp. 390-395. Disponible en: https://doi.org/10.1080/00963402.2016.1240476 (6-7-2017).

13 Para consultar en detalle y el alcance de estas promesas: Jos G. J. Olivier et al., Trends in Global CO Emissions: 2015 Report, PBL Netherlands Environmental Assessment Agency, The Hague, 2015. Disponible en: https:// goo.gl/pMJUV2 (6-7-2017). Y UNFCCC, Climate Action Now: Summary for Policymakers 2017, United Nations Climate Change Secretariat, Bonn, 2017. Disponible en: https://goo.gl/LfYRDw (6-7-2017). 
(2) Lograr un incremento en el alcance de los compromisos adoptados. Se trata de un reto que resulta crucial, pues tal como se encuentran las cosas ahora, aun cuando cada país llegase a cumplir sus promesas al pie de la letra, el objetivo de mantener el aumento de la temperatura promedio por debajo de los $2^{\circ} \mathrm{C}$ no podría alcanzarse ${ }^{14}$. Para que ello sea posible es necesario que las emisiones de $\mathrm{CO}_{2}$ experimenten "una reducción de entre el $40 \%$ y el $70 \%$ para 2050 en comparación con 2010, y niveles de emisiones próximos a cero o inferiores en $2100 " 15$. Estos niveles se encuentran abismalmente lejos de lo prometido en París. La propia Christiana Figueres, quien fuera la secretaria de la UNFCCC durante la Conferencia de París (2015), declaró ante la prensa que lo que en realidad había motivado la disposición de llegar a un acuerdo y el carácter de las promesas no tenían que ver con ninguna responsabilidad moral o con la intención del salvar al planeta, sino con el interés particular de cada país por su economía ${ }^{16}$. La franqueza aquí en las palabras de Figueres partía de exaltar el valor simbólico del acuerdo y la esperanza de que, a partir de él, se pudiera alcanzar en los años siguientes algún acuerdo más ambicioso y que se encontrase, este sí, más cercano a los objetivos planteados desde 2009 en Copenhague.

(3) Sobrevivir a una eventual salida de los Estados Unidos. El presidente Donald Trump, en concordancia con su política America First, anunció al mundo en junio de 2017 su intención de sacar a su país del acuerdo de París. Este acto ha constituido el colofón de lo que inició como una serie de pronunciamientos por parte de diversos actores políticos tanto dentro del partido republicano como dentro del círculo cercano al presidente, tendientes a descalificar o poner bajo cuestionamiento la veracidad científica del propio calentamiento global o, en el mejor de los casos, de su cualidad antropogénica, situación que ha propiciado una preocupante polarización política de la cuestión en aquel país ${ }^{17}$. Si bien es cierto que personajes como Emmanuel Macron y Xi Jinping han respondido asumiendo el liderazgo de la lucha en el plano internacional en contra del calentamiento global, la salida de un país como Estados Unidos, por lo que significa y por tratarse del segundo país más contaminante en términos de emisiones de $\mathrm{CO}_{2}$, puede terminar de echar por tierra un esfuerzo como el de París, que, no obstante, implica una contribución apenas incipiente a la causa del calentamiento global. "Don't take Trump literally, take him seriously". Se trata de una acertada frase que ha acompañado al ahora presidente de los Estados Unidos desde la campaña y describe casi a la perfección esta situación, ya que, según lo estipulado en el artículo 28 del acuerdo de París,

14 En ello coinciden: Mecklin, "It is Two and a Half Minutes to Midnight, 2017 Doomsday Clock Statement"; Paltsev, "The Complicated Geopolitics of Renewable Energy", pp. 390-395; y Reilly et al., Energy \& Climate Outlook. Perspectives From 2015.

15 IPCC, Climate Change 2014: Impacts, Adaptation, and Vulnerability. Part A: Global and Sectoral Aspects, pp. 11-20.

16 Sobre las declaraciones de Figueres: Mick Krever, "U.N. Climate Chief: Countries Acting for Economy, Not Planet": $C N N$ (1-12-2015). Disponible en: https://goo.gl/5KLw8k (6-7-2017); Michael Casey, "Can this Woman Convince the World to Act on Climate Change?": CBS (2-10-2015). Disponible en: https://goo.gl/DK4Rvt (6-7-2017).

17 Mecklin, "It is Two and a Half Minutes to Midnight, 2017 Doomsday Clock Statement". 
el plazo mínimo para que sea efectiva una retirada del mismo es de al menos cuatro años luego de su entrada en vigor. Por lo que la fecha más cercana en la que los Estados Unidos podrían estar abandonando el acuerdo sería el 4 de noviembre de 2020, un día después de la fecha en la que habrán de realizarse las próximas elecciones presidenciales. Es decir que, al menos por ahora, la intención del gobierno estadounidense de abandonar el simbólico acuerdo de París es a su vez simbólica (Don't take Trump literally). Pero es ese simbolismo el que puede provocar consecuencias letales en la lucha contra el calentamiento global, pues, a fin de cuentas, el gobierno de Trump pudo haberse limitado simplemente a incumplir sus promesas sin siquiera el temor a enfrentar sanciones. En cambio, ha decidido hacerlo parte elemental de su bandera política (take him seriously).

\subsection{Un contexto de desafío político}

"Los problemas del medio ambiente solo se pueden resolver mediante discusiones y acuerdos internacionales"18. Es por ello que, si de verdad se quiere reducir el uso de combustibles fósiles, resulta necesario contar con la (buena) voluntad de los gobiernos nacionales para regular o desincentivar su consumo. Pueden hacerlo, pues tienen a su disposición diferentes herramientas para ello que, de hecho, en algunos casos ya están siendo implementadas. Los ejemplos más claros de esto son los estímulos fiscales en favor del uso de energías renovables; los gravámenes fiscales encaminados a desincentivar la producción de combustibles fósiles, como es el caso del aclamado carbon tax o las regulaciones de carácter ambiental que directamente fijan límites de emisiones. Sin embargo, como se alertaba antes, lo que más dificulta la resolución o la mitigación de las amenazas globales es que no basta con que una nación por sí sola decida someterse a los dictados de la razón, es necesario que haya un esquema de cooperación a gran escala para que pueda haber esperanza.

Así pues, la única salida visible no se encuentra ni en el egoísmo ni en los mercados sino en la cooperación entre Estados. Pero la cooperación tiene por fuerza que pasar por la aduana de la política, y es precisamente desde esta realidad que la problemática que acarrean estas amenazas (todas) adquiere sus dimensiones reales. El caso del calentamiento global nos muestra el ejemplo paradigmático. Hoy que, aunque de manera incipiente, ha dado inicio la transición hacia las energías renovables, el statu quo del poder global que ha sido forjado en gran medida gracias a la importancia de los combustibles fósiles se ve amenazado. Por lo tanto, es lógico que en los grandes centros de poder, tanto del lado de la oferta (los países de la OPEC, Rusia, Estados Unidos) como incluso, del lado de la demanda (China, la Unión Europea, Estados Unidos de nuevo), los países se muestren reacios a aceptar sin reticencias la nueva era de la energía renovable. Y es que el hecho de que las nuevas fuentes de energía se encuentren mucho más diversificadas hace posible que aumente el número de actores involucrados en su producción. Además, el fomento y aprovechamiento de los recursos renovables depende de un nuevo tipo de circunstancias de carácter topográfico o climático que no necesariamente seguirán favoreciendo a aquellos que hasta

18 Ulrich Beck, La sociedad del riesgo, Paidós, Barcelona, 1998, p. 54. 
ahora han gozado de disponer de las mayores reservas de petróleo, gas y carbón ${ }^{19}$. En consecuencia, la estrategia parece estar encaminada a hacer que esta transición sea lo suficientemente paulatina como para que los ahora grandes protagonistas del juego geopolítico pierdan el menor poder posible en el proceso, aun si con ello ponen en riesgo el futuro de la humanidad.

La política es el sistema por el cual es posible y deseable explotar las vías de acción necesarias para aliviar el riesgo de que estas catástrofes sucedan y lograr alcanzar con ello cierto grado de seguridad. Pero mientras que las causas del peligro aquí descritas tienen un carácter eminentemente global, la política mantiene su centro de poder en los Estados, constreñido a los tradicionales limites espaciales de estos:

De lo que fundamentalmente se trata es de percibir cómo esta institución [el Estado], que siempre ha vivido por y para la territorialidad y el control se está viendo desbordada en estos dos ámbitos por efecto directo de la combinación entre los fenómenos de la mundialización y la complejidad creciente, que se encuentran en una relación de retroalimentación mutua. Una sociedad compleja, corporativa y plural y, por ejemplo, un sistema productivo y financiero que trascienden las fronteras, no se pueden someter hoy sin graves distorsiones dentro de límites territoriales circunscritos a un poder político concreto. La consecuencia es que este, o bien se ve obligado a renunciar a muchas de sus atribuciones tradicionales o debe replantearse drásticamente la forma en la que trata de imponerse sobre los diferentes campos sociales ${ }^{20}$.

Por otro lado, los intentos por establecer mecanismos de acción ejecutados fuera de estos límites estatales a través de organismos o acuerdos trasnacionales no han tenido la efectividad deseada. Si consideramos que el comienzo del auge de la globalización sucedió en el ocaso del sistema Bretton Woods, estaríamos hablando de que en más de treinta años la política no ha sido capaz de romper el cascarón y tener un papel más funcional en la escala global. No ha sido capaz de dejar atrás el viejo Westphalian frame.

\subsection{La acuciante necesidad de transformación de la política}

Si en 1650, la gran preocupación de Thomas Hobbes (1588-1679) a la hora de escribir el Leviatán consistía en la capacidad del Estado para garantizar la seguridad de los ciudadanos que lo conformaban; hoy, a menos de cuatro siglos de distancia, no solo la gran mayoría de los Estados no han sido capaces de garantizar la seguridad de sus ciudadanos ante los peligros surgidos desde su propio interior, sino que los leviatanes modernos se nos presentan ahora indefensos ante las más graves amenazas que se yerguen sobre los ciudadanos del mundo.

El 11 de septiembre de 2001 posiblemente haya marcado el momento en que el paradigma de la seguridad se transformó de manera definitiva. Si bien estos no fueron los primeros atentados terroristas, marcaron un antes y un después en la historia de la seguridad de los Estados. Fue a partir de entonces cuando se hizo patente el

19 Paltsev, "The Complicated Geopolitics of Renewable Energy", pp. 390-395.

20 Fernando Vallespín, El futuro de la política, Taurus, Madrid, 2000, pp. 106-107. 
riesgo que significan este tipo de organizaciones como amenazas transfronterizas que ya no funcionan bajo los clásicos códigos westfalianos. La yihad global fue capaz de hacer mella en la entonces nación más poderosa del mundo y que a la fecha sigue siendo la que más gasta en "defensa". Lo del " $9 / 11$ ", al igual que otros acontecimientos posteriores, fue un golpe de realidad para una política que funciona con una lógica cada vez más desfasada, un "repentino despertar en el extraño nuevo mundo de la sociedad del riesgo" 21 .

El paso del tiempo evidencia el hecho de que, a la hora de intentar prevenir este tipo de problemas, las medidas de talante estatalista han fracasado por completo en el largo plazo. Aun cuando muchas de estas sean implementadas con la firme convicción de sacrificar todos los medios necesarios en aras de alcanzar los fines trazados - el intervencionismo à la Monroe; el establecimiento de protectorados de facto como parte de una estrategia geopolítica heredada de la Guerra Fría; el convenio de escandalosos pactos de carácter económico o político con sanguinarios regímenes dictatoriales; o el intervencionismo militar en cualquiera de sus formas - no pueden seguir siendo moneda corriente en la resolución de conflictos o en el alivio de amenazas, mucho menos si se habla de problemas seguridad global como estos. Allí donde la miseria ha sido el mejor combustible a la hora de alimentar el odio y de fomentar una insoportable antagonía en contra de lo considerado occidental, es necesario implementar estrategias enfocadas verdaderamente en buscar el desarrollo de aquellos sitios en las que se gestan estos sentimientos. No es suficiente con "llevar la democracia" a una nación vejada y humillada por la supremacía militar de un grupo de potencias; es necesario, en primer lugar, garantizar una paz social (democrática o no) que sea viable y sustentable. Errores como los de Afganistán e Iraq no pueden seguir repitiéndose.

Esta realidad global tiene de particular que se inscribe en un proceso $^{22} \mathrm{o}$ en $u n a$ historia ${ }^{23}$ cuya principal característica es que no es posible definir con exactitud las condiciones de su sustancia ya que no ha sido culminado y sigue cambiando de manera constante. Además, es importante recalcar que este proceso no discurre sobre una sola vía, tampoco tiene efectos sobre un único ámbito de la vida social, "ni sigue una lógica, ni repercute por igual en las diferentes sociedades, grupos, empresas o sectores productivos". Si se entiende la globalización como la "progresiva "extensión' de las formas de relación y organización social, que desbordan los espacios tradicionales y se expanden hasta abarcar el mundo entero"24, queda claro por qué lo global es más que la suma total de las relaciones transnacionales ${ }^{25}$ y es posible, en consecuencia, hablar de una globalización de los riesgos, de la economía, de la cultura, etc.

Esta circunstancia implica también que no todos los campos de la actividad humana se globalizan al mismo ritmo, algunos como es el caso de la política, se man-

${ }_{21} \quad$ Ulrich Beck, World at Risk, Polity Press, Cambridge, 2009, pp. 67-68.

22 Probablemente quienes mejor han abordado el tema de la globalización como un proceso son: Roland Robertson, Globalization: Social Theory and Global Culture, SAGE Publications, London, 1992; y Martin Albrow, The Global Age: State and Society Beyond Modernity, Polity Press, Cambridge, 1996. Sin duda, dos de los principales teóricos de la globalización.

23 Pierre Rosanvallon, Por una historia conceptual de lo político, Fondo de Cultura Económica, Buenos Aires, 2003, passim.

24 Vallespín, El futuro de la política, pp. 30-31.

25 Albrow, The Global Age, p. 120. 
tienen rezagados. Y es que, como se mencionaba arriba, ha sido muy poco lo que esta ha cambiado desde el inicio de las mundializaciones, desde el inicio de esta realidad global. El proceso de renovación ha sido principalmente subpolítico, quedando a cargo de la economía, la ciencia y la tecnología; ámbitos de carácter autorreferencial (Niklas Luhmann,1927-1998) que no se rigen bajo mecanismos democráticos, se trata de una subpolítica que "ha quitado a la política del rol dirigente de la configuración social" 26 .

Se está contemplando pues, el paulatino triunfo de lo que Pierre Rosanvallon llama la "contrademocracia impolítica"27. Es por ello que ahora, cuando en palabras de Carl Schmitt (1888-1985) las amenazas globales se nos presentan como el enemigo óntico del ser humano, queda demostrada la negligente lentitud de la política para adaptarse a los últimos procesos de transformación; y tendríamos que empezar a hablar, sin que se nos acuse de alarmistas, de que hemos entrado a un periodo de emergencia y de que hay acciones que tienen que ser emprendidas cuanto antes. Pues si hasta hace unos años el riesgo seguía consistiendo en un retorno al estado de naturaleza, de la guerra de todos contra todos, hoy, ya no nos quedaría ni eso, y nos estamos acercando al punto de no retorno.

Sin embargo, para que todo esto mantenga cierto sentido es necesario contar con un tanto de optimismo y pensar que, con la urgencia como sustituto de un verdadero framework de cooperación internacional, será posible implementar las suficientes medidas contingentes como para que la humanidad pueda "salir del paso" del problema del calentamiento global. Pero estas medidas contingentes tendrían que ser entendidas como lo que verdaderamente significan: una manera de ganar tiempo valioso. No pueden ser utilizadas solamente para posponer lo que hoy, de acuerdo con las categorías de Nick Bostrom, representa un "riesgo existencial" 28 . Y es que aun cuando el sueño de la ingeniería ambiental se llegase a hacer realidad y se perfeccionasen los milagrosos procesos de captura y almacenamiento de dióxido de carbono de tal manera que el problema del calentamiento global pudiera ser arreglado como por arte de magia ${ }^{29}$, es probable que, en el futuro, sea otro problema el que encienda las alarmas de la aniquilación mundial mientras la impávida política, ejercida a través de la clásica constelación de naciones, planee una estrategia cuyos cálculos de riesgo se encuentren, todavía, cuantificados en votos.

Es por ello que, llegados a este punto, parece que el principal problema global paradójicamente no tiene su origen en la globalización misma, sino en la falta de una adecuada globalización política.

26 Beck, La sociedad del riesgo, p. 20.

27 Para más sobre las formas de lo subpolítico: Pierre Rosanvallon, La contrademocracia: La política en la era de la desconfianza, Manantial, Buenos Aires, 2007. Habría que considerar rebautizar este concepto como "contra-contrademocracia".

28 Nick Bostrom, "Existential Risk Prevention as Global": Global Policy, vol. 4, n. ${ }^{\circ} 1$ (2013), pp. 15-31.

29 Leo Hickman, “Timeline: How BECCS Became Climate Change's 'Saviour' Technology”: Carbon Brief (13-42016). Disponible en:https://goo.gl/XwkgfU (6-7-2017). 


\section{Hacia una globalización política}

\subsection{El Estado como el núcleo del proceso de transformación de la política}

La cuestión ahora radica en preguntarnos el cómo de una globalización política. Deberíamos de ser capaces de encontrar un camino que plantee una salida funcional: viable y sustentable; uno a partir del cual nos sea posible dejar atrás aquellos rasgos que más lastran el proceso de redefinición de lo político pero que, al mismo tiempo, nos permita conservar lo construido hasta ahora. Tendríamos que ser capaces, además, de resolver una de las cuestiones más fundamentales al hablar de una globalización política: la pertinencia del Estado. ¿Es esta forma de organización política más que una estructura obsoleta, un cascarón que nos impide crecer? ¿Es que acaso no hay política deseable más allá del Estado? ¿Hay una sola respuesta o un solo planteamiento que seguir respecto de un proyecto de globalización política? “¿Estamos ante el fin del Estado y su sustitución - a su debido tiempo - por una forma de organización política distinta o ante un 'nuevo' Estado en evolución?"30

Hoy, que seguimos postrados frente a la misma duda, parece que el fin del Estado o una profunda evolución de este son procesos que no han iniciado a gestarse y que no lo harán, al menos en el futuro previsible. Es posible que la globalización de los ámbitos sociales nunca llegue a transformar a los Estados. ¿En qué medida podrían transformarse como para que dejasen de ser Estados? Por otro lado, sí es posible observar cambios derivados de un proceso globalizador en la política y, aunque estos no sean lo suficientemente importantes como para considerar que la política se encuentra en posibilidad de hacer frente a los problemas globales mencionados antes, la evidencia de que ha iniciado un proceso de transformación en este ámbito hace pensar que serán las formas de este proceso las que determinarán el futuro del Estado y no al contrario. Prueba de ello es que los cambios que la figura del Estado ha sufrido en esta época no tienen que ver con su estructura, sino que, tal y como se acusaba en párrafos anteriores, tiene que ver con una reducción en la capacidad de cumplimentar sus funciones. Esto "aplica para todas las funciones clásicas del Estado, desde la salvaguarda de la paz y de la seguridad física hasta la de garantizar la libertad, el estado de derecho y la legitimación democrática"31 y podríamos ir incluso más lejos en esta enumeración de transformaciones si tomamos en cuenta lo que Arjun Appadurai llama ethnoscapes; es decir, si tomamos en cuenta que la globalización de las identidades ha comenzado a erosionar, por su parte, la capacidad del Estados de configurar su propio demos ${ }^{32}$.

Hay que decir que un proyecto de globalización política no constituye un objetivo que los Estados, como tales, no puedan alcanzar. En sentido amplio se puede decir que siempre ha existido una política en el nivel global, la propia Paz de Westfalia puede ser un ejemplo de ello. La diferencia es que el proceso de globalización, de desbordamiento de los espacios tradicionales que experimenta la política ahora, implica un cambio de paradigma respecto de su esfera de repercusión. No se trata tanto de la política que sucede fuera de los Estados, sino del reconocimiento de una

\footnotetext{
Vallespín, El futuro de la política, p. 91.

Jürgen Habermas, Europe: The Faltering Project, Polity Press, Cambridge, 2009, p. 91.

Arjun Appadurai, Modernity at Large: Cultural Dimensions of Globalization, University of Minnesota Press, Minneapolis, 2009, pp. 27-47.
} 
política que afecta a todos los Estados. El post-Westphalian frame exige tener claro que, en un mundo globalizado, un tratado multilateral afecta también a las naciones no firmantes. Por lo que las tensiones entre el viejo paradigma y la nueva realidad aumentarán a medida que, a su vez, aumenten los actores internacionales (no estatales), se agraven los problemas globales ${ }^{33}$ y los Estados, que a pesar de todo se mantienen como los principales actores en la dimensión global, sigan actuando conforme a sus propios intereses, atendiendo solo al interior de sus fronteras y sin preocuparse demasiado por las consecuencias que ello pueda acarrear en una escala internacional. Es por ello por lo que los Estados habrán de mantenerse como puntos de referencia a la hora de hablar de un proyecto de globalización política, así sea para proyectar su extinción.

Entre los muchos planteamientos sobre el futuro de una política global, probablemente uno de los más comunes sea aquel que concibe la creación de una unidad política que abarque el total de los países en el mundo: bajo la forma de una comunidad tejida en torno al concepto de lo humano en la que la existencia de los Estados-nación no tendría cabida; o mediante el forjamiento de una sociedad global erigida al amparo de una organización mundial que se mantenga o bien por encima de los Estados, o bien los organice como partes de una federación o confederación global. Roland Robertson considera que este tipo de propuestas surgen a partir de diferentes respuestas a la globalización y las clasifica en dos rubros principales en función de lo que podríamos llamar una adscripción más o menos comunitarista: Global Gemeinschaft y Global Geisellschaft ${ }^{34}$. Así pues, ya sea que se proyecte la desaparición de los Estados en favor de una única entidad supranacional absoluta o solo se los pretenda someter a esta, pensar en ese ente político total es algo que parece, quizá hoy más que nunca, demasiado desapegado de la realidad. Hay múltiples consideraciones que fomentan el escepticismo en este sentido; la propia disposición de las naciones en torno a un proyecto de tales características ya suena demasiado irreal, sobre todo en cuanto a la conformación de un demos global se refiere. Y es que no solo hay que tener en cuenta la gran cantidad de insalvables conflictos entre países que ya hacen imposibles las relaciones bilaterales entre ellos. Además, esta idea nos obliga a sopesar los aspectos de carácter ideológico, cultural o religioso que entrarían en contradicción con un proyecto planteado desde el "etnocentrismo occidental".

Un planteamiento de estas características tendría que presuponer una revisión del estado en el que se encuentran las relaciones de occidente con "cada uno de sus otros". Mientras que las diferencias, principalmente con el islam, parecen hacerse cada vez más ríspidas, los valores de Occidente, pretendidamente universales, siguen siendo valores a universalizar; $y$, si no parece haber una estrategia muy clara respecto a cómo lograr tal cometido, mucho menos se puede visualizar por dónde sería posible comenzar a construir las bases de una nación que las abarque a todas, mucho menos las de una única comunidad humana. Que se esté padeciendo un desajuste entre los problemas globales y la medida en que la política es incapaz de superar sus límites tradicionales no implica que sea necesario ampliar los límites espaciales de las unidades políticas hasta crear una sola absoluta y definitiva. Se trata más bien, al

James N. Rosenau (1924-2011) denominó esta situación como “turbulencias” en James N. Rosenau, Turbulence in World Politics. A Theory of Change and Politics, Princeton University Press, Princeton, 1990.

34 En total son ocho las formas que adoptan las respuestas de la globalización dentro del esquema de Robertson en Robertson, Globalization, pp. 78-79. 
menos por ahora, de dotar de capacidad a la política para que pueda actuar fuera de las fronteras de los Estados en un ámbito de aplicación común.

Es posible que la idea de una nación o comunidad absoluta sea la culminación del proceso de globalización política, pero ahora que nos encontramos en una primera instancia de ese proceso de postnacionalización caracterizado por (1) el surgimiento de nuevas formaciones que aglutinan la lealtad de aquellas identidades que se encuentran dispersas; (2) el surgimiento de nuevas formas de organización a partir de las cuales regular el tráfico de ideas y recursos a escala global y (3) la proliferación de formas nacionales que buscan romper las limitaciones territoriales en las que se encuentran inmersas ${ }^{35}$; los Estados, como las esferas de organización política que son, continúan siendo un instrumento elemental de este mismo proceso, aun si de lo que se trata es de hacerlos desaparecer.

\subsection{La económica, la globalización por excelencia}

El actual marco de negociación de carácter transnacional que funciona a través de tratados y convenciones que firman los países, como parte de las relaciones bilaterales entre estos o como parte de su participación en diferentes organismos y estructuras internacionales, es probablemente el más desarrollado y explotado proyecto de globalización política hasta el momento. Sin embargo, estamos hablando de un marco de negociación que sigue regido por la lógica westfaliana de toda la vida y los acuerdos a los se llega en este, en la mayoría de los casos, dejan fuera a otros importantes actores del terreno global como es el caso de los grandes poderes económicos que, no obstante, toman otras vías y estrategias para hacer valer el peso de sus intereses. Por ello, de nada serviría que se firmase un acuerdo en el que las naciones más poderosas se comprometieran a regular el nivel de emisiones de contaminantes en el interior de sus fronteras si, al mismo tiempo, el modus operandi de las grandes empresas sigue consistiendo en colocar fábricas en países en vías de desarrollo con el fin de aprovechar los bajos salarios, las muy limitadas prestaciones laborales y las laxas leyes ambientales del país en cuestión. Pues como se ha visto antes, tratándose de calentamiento global, poco importa dónde se ubiquen las fábricas.

Gracias al dinamismo que ha traído consigo la globalización económica, cualquier empresa que esté en capacidad de permitírselo puede alcanzar ganancias exorbitantes si produce en países en desarrollo, vende sus productos mundialmente y cotiza en las principales bolsas de valores del mundo. Sin embargo, esta forma de hacer negocios genera diversas complicaciones que se añaden a las ya expuestas en el escenario global, pues incentiva a los países en desarrollo a buscar atraer a la inversión extranjera al punto de estar dispuestos a hacer del dumping en cuestiones ecológicas, laborales y financieras el centro de su estrategia. Y aunque es verdad que este esquema de las cosas aporta, al menos en el corto plazo, beneficios a todas las partes involucradas, estos son altamente desiguales. En el caso de los países en vías de desarrollo, se pretende alcanzar cierto nivel de crecimiento que permita la inversión en infraestructura con el fin de atraer más inversión. Tristemente, el crecimiento al que llegan estos países en muy pocas ocasiones se traduce en una mejora en la calidad de vida o en las garantías

35 Appadurai, Modernity at Large, pp. 48-50. 
sociales de los ciudadanos; lo impiden la existencia de oligarquías, la corrupción endémica y la propia incompatibilidad que esto tiene con el concepto de "competitividad". Para el caso de los países que el Fondo Monetario Internacional denomina "economías avanzadas", el beneficio llega por una doble vía: la primera consiste sobre todo en la apertura de mercado para los productos que ellos mismos producen, principalmente vehículos, maquinaria, equipo médico, fármacos, equipamiento electrónico, combustible y armas; la segunda vía es la de los mercados financieros, a partir de los cuales se obtiene un beneficio del crecimiento de las compañías que explotan el dumping de los países en desarrollo. Así pues, mientras la situación es de ganar-ganar para los pocos que venden cara la membresía a su selecto grupo, los que buscan alcanzar un mayor nivel de desarrollo, que son la inmensa mayoría, compiten por lo bajo, unos con otros, en la famosa race to the bottom.

Es por ello por lo que no es posible no hacer notar el hecho de que algunos rasgos del esquema plasmado por Immanuel Wallerstein en su economía-mundo durante los años ochenta se mantienen vigentes. "El intercambio desigual de bienes y servicios, de tal forma que, en la mayor medida posible, aquella plusvalía extraída de las zonas periféricas de la economía-mundo sea trasladada a las zonas centrales" es un factor que, de la mano con el imperativo del libre comercio, preserva la hegemonía de los países desarrollados a la vez que empuja a los otros a caer en las estrategias de catching-up ${ }^{36}$.

Nadie obliga a los países en desarrollo a participar de este sistema y hacerles el juego a las grandes potencias económicas, se podrá objetar, pero ¿hay alternativa? Quizá vayan demasiado lejos quienes claman que lo que se vive actualmente no es más que una actualización del sistema imperialista de antaño, pero lo cierto es que hay una similitud evidente. Muchos países de los llamados en desarrollo no tienen otra alternativa que adaptarse a las demandas dictadas por los países que representan para ellos sus mayores mercados. Y es que no se puede entrar en el mercado global solo un poco, se tiene que entrar de lleno "y a lo loco". Así lo prescribe el Consenso de Washington, al que todos los Estados han de adscribirse tal como amablemente receta el FMI, pues en el mercado global del libre comercio regido por la OMC, la única libertad que al parecer tienen los Estados es la del libre comercio.

En esta economía post-Bretton Woods, como apunta Dani Rodrik, los Estados han perdido un importante espacio de maniobra: el que les permitía implementar políticas con la finalidad tanto de fomentar como de proteger de las presiones de los mercados externos la propia industria y ciertos sectores productivos clave. Lo que pasa es que ahora el establecimiento de aranceles especiales sobre bienes de importación, de subsidios en favor de una única industria o sector, o cualquier medida que pueda ser considerada discriminatoria o que viole el principio de la nación más favorecida (MFN), se encuentran proscritos por la Organización Mundial de Comercio. $\mathrm{Y}$ aunque dentro de la reglamentación de esta organización hay una consideración especial en favor de los países en desarrollo y ciertos casos de excepción, muchas de estas naciones experimentarían mejores resultados fuera de los estrictos esquemas de este libre comercio a rajatabla, donde pudieran implementar medidas más acordes con su situación particular y no una mera apertura indiscriminada ${ }^{37}$.

\footnotetext{
36 Immanuel Wallerstein, The Politics of the World-Economy, Cambridge University Press, Cambridge, 1984, pp. 54-55.

37 Dani Rodrik, The Globalization Paradox, Norton, New York, 2011, pp. 159-184.
} 
Resuenan las palabras de Figueres cuando señalaba que los países firmaban el acuerdo de París pensando cada uno en su propia economía. Por momentos parece que toda aspiración por una política global ha sido copada por las consideraciones de carácter económico de los Estados y si no hay espacio para otro tipo de planteamientos en el terreno global, no hay duda en que las complicaciones para una política atrapada en la westfalia no hacen más que aumentar. El sistema capitalista global, cuya realización depende de la búsqueda permanente de producir y crecer, de la ambición por el poder a través del dinero o de la fuerza y de las condiciones de vida que este sistema ha impuesto sobre los ciudadanos "menos afortunados", tiene una estrecha relación con cada uno de los problemas globales aquí mencionados: contaminación, competencia armamentística, organizaciones terroristas, organizaciones criminales; $y$, a todo esto, tendríamos que añadir migración. Hablamos de problemas globales que por globales no son menos discriminatorios, pues mientras las riquezas se acumulan arriba, los riesgos se acumulan abajo, justo donde la vulnerabilidad a los mismos es mayor ${ }^{38}$. Sin embargo, y pese a que a alguno le pudiera parecer lo contrario, no se pretende trasladar la decimonónica lucha de clases a una escala global y dotar de nueva vida a cierta idea de alternativa al capitalismo, ya sea esta reciclada o replanteada. Pues, aunque alguna variante del marxismo hubiese tenido en algún momento en mente la implementación de un sistema político global de carácter supranacional, hoy, a más de un cuarto de siglo desde la caída del muro de Berlín, somos testigos de cómo el capitalismo se pudre de éxito y de cómo este último parece formar tanto parte de nosotros mismos que la posibilidad de aplicar cualquier alternativa se nos muestra como una empresa tanto más difícil que la de formar un Estado que los abarque a todos.

\subsection{La Unión Europea, un experimento globalizador}

Aun cuando el ideal romántico del fin del capitalismo pudiera parecer demasiado lejano como para plantear a partir de él una posible alternativa al cómo de la globalización política, sigue habiendo esperanza. Probablemente, la mejor respuesta que han podido dar los países a los problemas globales ha sido la creación de organismos transnacionales a los que han encomendado diversas tareas muy específicas; tal es el caso de la Organización de las Naciones Unidas y sus respectivas agencias especializadas, el Fondo Monetario Internacional, el Banco Mundial y la Organización para la Cooperación y el Desarrollo Económicos.

Por supuesto, estos esfuerzos no han estado exentos de vicios y debilidades. A lo largo de los años se ha podido constatar cómo esta asignación de tareas también ha venido acompañada de una cierta delegación de responsabilidad por parte de los Estados que, en muchas ocasiones, llegan al punto de actuar en contraposición de los dictados emanados por estos organismos, aun cuando sigan "formando parte" de ellos y continúen aportando en su financiación. La falta de una potestad mayor o de atribuciones de carácter vinculante y la excesiva burocratización de su funcionamiento han impedido en gran medida que estos organismos alcancen metas más significativas. Y pese a que seguramente con su creación se habrán alcanzado

38 Beck, La sociedad del riesgo, pp. 40-41. 
importantes logros y mejorado con ello la vida de una incontable cantidad de personas, es claro que su participación en el ecosistema político global no ha gozado del suficiente impacto como para contrarrestar los efectos que la vorágine capitalista ha tenido sobre el medio ambiente y el subdesarrollo.

Frente a este tipo de organismos que funcionan como actores de política internacional ajenos a los Estados que los auspician está la Unión Europea que, así sea catalogada como un organismo o como una comunidad política, es una entidad que realmente ha sabido colocar su campo de acción sobre los países que la integran. Lograr la aplicación de normativas comunes en tal cantidad de países, algunos tan dispares como Francia y Estonia, no ha sido una tarea fácil de alcanzar, y se han necesitado muchos años y el desarrollo de una sofisticada red de instituciones y organismos que operan de manera conjunta para procurar tanto su buen funcionamiento como también la integración entre sus miembros.

A pesar de lo anterior, la legitimación democrática, uno de los principales objetivos políticos de la UE, ha trascendido como el aspecto más problemático para este organismo y hoy en día amenaza con volverse su punto de quiebre ya que no se ha logrado establecer una identidad comunitaria europea sólida sobre la cual establecer una esfera pública que satisfaga este imperativo político. Y es que, si bien la UE ha tenido el tino de no fundamentarse únicamente sobre una serie de prerrogativas económicas, sino que ha buscado hacerlo, además, sobre la base de un entendimiento común y del establecimiento de un conjunto de principios político-culturales - fundamentalmente la democracia, los derechos humanos, la multiculturalidad, la libertad, la igualdad y la cooperación-, no ha sabido postrarse como el elemento de salvaguarda de los mismos. Toda vez que las cuestiones relativas a hasta qué punto y cómo se cumplen estas disposiciones de carácter sustantivo siguen formando parte de la soberanía de cada una de las naciones, la calidad de la democracia o el nivel de respeto a los derechos humanos son elementos que no resultan tan homogéneos como se querría dentro de los países miembros. No obstante, esto resulta entendible si se tiene claro que este organismo no es un gran Estado que haya surgido de la fusión de otros más pequeños; sino en todo caso, se trata de una estructura política surgida de una serie de acuerdos firmados por los países miembros, sujetos de derecho internacional que, como tales, mantienen cada uno una soberanía propia. En consecuencia, la Unión Europea más allá de garantizar la "libre circulación de personas" entre algunos de los países miembros y de homologar ciertas disposiciones normativas de segundo orden, es un ente cuya importancia mayor radica, a fin de cuentas, en lo económico.

Al no existir una verdadera identidad europea, el único elemento que realmente une a los europeos es el euro. La moneda, contante y sonante como es, une de facto el destino de los ciudadanos. Y aunque cada país posee su propio patrimonio y una economía particular, no hay momento que haga brotar con más energía el sentimiento de pertenencia a Europa que cuando el Banco Central Europeo decide desembolsar una cantidad inconmensurable de dinero en pos de rescatar a algún país en desgracia o cuando ese país rescatado es al que uno pertenece y, en este caso, el problema radica en encontrar la forma de pagar el prestamo (o de no pagarlo). Es por ello por lo que no sorprende que cuando hay momentos de crisis o de políticas de austeridad es cuando más crece el llamado euroescepticismo. Europa muestra su faceta más hostil, y entre sus ciudadanos comienzan a surgir expresiones que en muchos casos contravienen los principios sobre los que en el papel se encuentra fundada la unión, debilitando aún más los lazos que la mantienen unida. 
Resulta paradójico que la principal crítica que se hace a la Unión Europea sea que no es lo suficientemente democrática, cuando han sido precisamente en las elecciones al Parlamento europeo donde los partidos más euroescépticos y menos democráticos han tenido su mayor éxito. En un principio era común escuchar la explicación de que esta circunstancia se debía precisamente a la percepción de un déficit democrático. No obstante, mientras que la Unión Europea no se ha vuelto más democrática, la popularidad de una prototípica serie de partidos radicales y populistas sí se ha incrementado considerablemente en la mayoría de los países miembros y ya no solo en cuanto a lo que a las elecciones europeas se refiere. La Unión Europea es, además, un gran ejemplo de lo que Rosenau llama las dinámicas de la "fragmegración", las cuales denotan cómo cada tendencia globalizadora da origen a una localizadora y viceversa; en este caso, "cada propuesta encaminada a buscar una mayor integración ha sido respondida por intensos esfuerzos de parte de algunos de los gobiernos y comunidades afectadas, por adoptar políticas diseñadas para preservar prerrogativas y practicas locales" 39 .

\subsection{El fin de la humanidad}

Con todas las deficiencias o fallas de diseño de la Unión Europea, resulta hasta cierto punto inadecuado señalar a esta organización como la única responsable de lo que ocurre en cada uno de los países miembros, pues como se ha dicho, estos mantienen su soberanía en gran medida y en la mayoría de los aspectos de relevancia. Pues aun dentro de los lineamientos establecidos por los tratados firmados como parte del marco de los acuerdos de la UE, mantienen la potestad de decidir por sí mismos las políticas a aplicar en temas fiscales, laborales, educativos, económicos, etc. En consecuencia, es posible afirmar que, en gran medida, la creación de una identidad europea fundamentada en los principios mencionados antes tiene que ver con la falta de una mayor integración social de estos principios dentro de los propios países miembros.

Supongamos que las principales potencias económicas se comprometieran verdaderamente con el medio ambiente y el respeto a los derechos humanos (ya no digamos con la cooperación activa con el desarrollo de otros países), de tal manera que estuviesen dispuestos a limitar el uso de combustibles fósiles y a acabar con el dumping económico y social descrito antes. Si sometieran la decisión a un referéndum, ¿los ciudadanos votarían a favor de estas medidas aun a sabiendas de que ello implicaría un deterioro en su calidad de vida? Esta pregunta hipotética dista mucho de ser a la vez retórica, no hay una respuesta clara ni es aquí un objetivo el estar en condiciones de saber cuál podría ser esta. Lo que sí está claro, respecto de los hipotéticos resultados que tendría este referéndum, es que, si bien la balanza pudiera no encontrarse ya inclinada hacia una respuesta negativa, en los últimos años hemos sido testigos de cómo la tendencia apunta hacia esa dirección. A veces pareciera que las sociedades, en general, han experimentado tal sobrecarga de globalización que han buscado retornar de lleno al viejo state-centric world $^{40}$, tratando de resguardarse así en toda la certidumbre que los Estados nación puedan llegar a otorgarles.

\footnotetext{
39 James N. Rosenau, The Study of the World Politics, Volume 2: Globalization and Governance, Routledge, London, 2006, p. 36.

40 Rosenau, Turbulence in World Politics, pp. 243-296.
} 
Desde finales del siglo pasado se ha señalado cómo el factor identitario se hacía cada vez más importante. Esto fue confirmado años después en la voz de figuras como Angela Merkel y David Cameron cuando anunciaron el fracaso de la visión multiculturalista en sus países. Ha sido justamente en las sociedades de mayor diversidad étnica, o en las que han experimentado mayor inmigración, en las que lo común no ha sabido anteponerse a lo diferente, sino que ambas cualidades se han engarzado en una dinámica de canibalismo mutuo ${ }^{41}$ que ha terminado por potenciar los conflictos de carácter identitario. Y es que por más chocantes que nos puedan resultar a veces los diagnósticos postmodernistas, lo cierto es que el salto hacia atrás que de manera generalizada parece haber dado el pensamiento de las sociedades occidentales es algo que resulta cada vez más constatable. Algo similar ha pasado con la cultura, que, frente a la apertura de una inmensa multiplicidad de oportunidades culturales que ha traído el avance en las tecnologías de la información, los usuarios han optado por aferrarse a los clásicos referentes locales, o por dejarse llevar por el mainstream dictaminado desde los Estados Unidos. Hoy más que nunca parece que el único progreso al que puede aspirar la humanidad es a uno que pueda traducirse a partir de la tasa de crecimiento del PIB, o ya ni eso.

Si se observan los resultados electorales y las políticas implementadas en los últimos años en los países con los más altos niveles de educación, bienestar y desarrollo en Europa, se puede apreciar con facilidad que, en ellos, también hay un creciente distanciamiento con los ideales de la Unión Europea, los cuales son en realidad meras recapitulaciones de los ideales de la Ilustración. Estos países que en algún otro momento no dudaríamos en señalar como los mejores exponentes del progreso de la humanidad, estas "sociedades fuertes", hoy son, sin embargo, los mejores exponentes del desencanto. Resulta desconcertante intentar explicar las razones de por qué el desarrollo económico, tecnológico y científico no se encuentra acompañado a su vez por un desarrollo moral, o si se quiere, político. Educación, calidad de vida, seguridad social, libertad, riqueza, distribución de la riqueza, transparencia, bajos niveles de corrupción y hasta calidad de la democracia; toda una serie de aspectos relacionados con el ejercicio del poder público susceptibles de ser cuantificados y que son utilizados para hacer comparaciones entre naciones, les otorgan de manera generalizada a ciertos países del norte y centro de Europa, y a sus habitantes, una posición privilegiada. ¿Cuál es la combinación adecuada de parámetros o el nivel de desarrollo necesario para lograr llegar al punto de ver desterrada de un país toda acción popular encaminada a echar abajo aquello que el ideal ilustrado y la democracia ha construido durante décadas? Es en estas naciones en las que menos se esperaría que aconteciese un considerable crecimiento en los votos a partidos populistas con discursos que van desde el nacionalismo a la xenofobia y cuya afiliación democrática es, por decir lo menos, muy cuestionable.

No hay nada realmente construido dirían algunos. Se trata solamente de una farsa conjugada alrededor de la hipócrita persecución de cierto ideal político que en el fondo únicamente pretende preservar las viejas estructuras de poder económico de toda la vida. La sociedad burguesa actual se encuentra condenada a derrumbarse sobre su vacío contenido y por el propio peso de sus contradicciones. La estrategia de los partidos antidemocráticos está montada sobre la propia lógica bajo la que funciona la alienada sociedad capitalista. Donde muchos quieren ver la antítesis del

${ }^{41}$ Appadurai, Modernity at Large, pp. 39-40. 
ideal burgués, lo que enfrentan, si es que en verdad lo enfrentan, es en realidad un virus engendrado dentro del propio sistema capitalista, que se aprovecha de las fallas de este para utilizarlas a su favor.

Lo que distingue a los demócratas de los antidemócratas no radica en una pretendida afiliación bondadosa de los ideales occidentales sino una cuestión de indiscreción: donde los primeros hacen una explotación más o menos controlada de las sociedades capitalistas y hacen lo posible porque esta se mantenga sustentable, los segundos van demasiado lejos, no se atañen a las reglas de este juego de caballeros oligarcas y en su hambre de poder están más que dispuestos a comerse hasta las plumas de esa gallina que pone huevos de oro. No hay nada construido alrededor del ideal ilustrado más allá de una gigantesca red de poder acumulado en unas pocas manos. Generaciones y generaciones de excesos, lujos y depravación cimentadas sobre siglos de injusticia y hambre.

No obstante, si se quiere apelar a un poco de principio de certidumbre, no es posible cerrarse en banda, proclamar una vez más el fin de la humanidad y señalar, de esta manera encarnizada, que toda presunción de progreso no es más que una farsa o una ilusión inocente. Por más desencanto por la sociedad que podamos recabar, es un hecho que los progresos tecnológicos que la humanidad ha alcanzado desde el auge de la revolución industrial han ayudado a mejorar la vida de los individuos, incluso las de aquellos más desamparados. Esto, por supuesto, no se ha dado en la forma en que todos quisieran, ni mucho menos ha cumplido con las expectativas depositadas en este desarrollo o en la llamada tercera ola de la democratización. Pero ver las cosas en términos absolutos y culpar de todo al capitalismo, a las globalizaciones o cualquiera que sea el siniestro personaje de moda, no nos ofrece ni siquiera el atisbo de una posible solución.

\subsection{De vuelta al Estado}

Después de todo, no parece muy plausible pretender que un organismo como la Unión Europea, por más que sea la mejor expresión de globalización política con la que contamos en la actualidad, cumpla con los imperativos de justicia que pudieran ser expresados desde una renovada teoría de la esfera pública adaptada a la realidad global actual, sobre todo si consideramos la cantidad de cabos sueltos derivados de ello.

¿Es posible que las esferas de lo público hoy, ofrezcan suficiente eficacia a la opinión pública como para limitar a los varios poderes que determinan las condiciones de vida de sus interlocutores? Y si lo es, ¿cómo? ¿Qué tipo de cambios (institucionales, económicos, culturales y comunicativos) serían necesarios tan solo para imaginar que las esferas de lo público puedan tener un rol genuinamente crítico y democratizador bajo las circunstancias actuales? ¿Dónde están los poderes soberanos que la opinión pública debería estar limitando en este momento? ¿Qué públicos son relevantes para qué poderes? ¿Quiénes son los miembros relevantes de un determinado público? ¿En qué lenguajes y a través de qué medios pueden comunicarse estos? ¿Y a través de qué infraestructura comunicativa ${ }^{42}$.

42 Nancy Fraser, "Transnationalizing the Public Sphere: On the Legitimacy and Efficacy of Public Opinion in a Post-Westphalian World", en Nancy Fraser et al., Transnationalizing the Public Sphere, ed. de Kate Nash, Polity Press, Cambridge, 2014, pp. 26-27. 
Ante lo anterior, cabría cuestionarnos además cuántas de las naciones europeas son o fueron capaces de cumplir cabalmente con los requisitos para la legitimidad y la eficacia de la opinión pública en estos términos, aun si consideramos únicamente la era westfaliana. A pesar de que la propia Nancy Fraser ofrece algunas "pistas" sobre cómo resolver el cómo de una globalización política: "el reto es doble: de un lado, crear nuevos poderes públicos transnacionales; del otro, hacerlos responsables ante las nuevas esferas públicas transnacionales" ${ }^{\text {"33; }}$ no parece nada claro cuál será el motor que posibilite la alineación de las múltiples voluntades e intereses de carácter nacional-westfaliano. Más aún, no parece claro cómo fomentar en la gran mayoría de ciudadanos un sentido de solidaridad tal que los haga estar dispuestos a sacrificar su calidad de vida en favor otros ciudadanos ubicados en otro lado del mundo, con los que no mantengan comunicación alguna, ni compartan geografía, cultura, religión o historia. Ni parece claro cómo hacer conscientes a los ciudadanos de la necesidad de una esfera pública global, por más instituciones y poderes públicos que, operando a escala transnacional o supranacional, sean responsables ante una "opinión pública" global. ¿Cómo lograr en los ciudadanos todo lo anterior si todavía hay una gran cantidad de ellos que no están muy convencidos de conceptos como la democracia, los derechos humanos, la multiculturalidad, la libertad, la igualdad y la cooperación?

Las ensoñaciones cosmopolitas de autores como David Held tampoco dan respuesta alguna a este tipo de cuestionamientos, ni siquiera aquellas pensadas para el "corto plazo". Y aunque este autor acote que "el hecho de exponer los objetivos de un modelo cosmopolita de democracia no implica que se crea que todo ello pueda ser alcanzado inmediatamente, ipor supuesto que no!" "44, el alejar de tal manera el foco de la atención sobre los problemas de la convivencia global actual hace que este tipo de modelos terminen resultando ser meros ejercicios de ingeniería institucional. Antes de plantear la construcción de organismos supranacionales encaminados a solventar el déficit de globalización política (déficit de poder) y el clásico déficit de legitimación de lo político, es necesario finalizar la construcción de las que serían sus unidades constitutivas y volver al Estado con el objetivo de culminar de una vez por todas con ese proyecto político.

Considero que el Estado no está completamente inventado aún, que no hay Estado que haya llevado los ideales de la ilustración hasta sus últimas consecuencias y tanto gobierno como sociedad rijan sus días a partir de ellos. Por supuesto, un mundo repleto de Estados perfectos no es una perspectiva poco ambiciosa, sin embargo, cuando aquí se habla de culminar el proyecto de Estado, se tiene en mente un esquema de mínimos. Dicho esquema dependería tan solo de un verdadero reconocimiento de los derechos humanos como norma primordial. Pero, siguiendo el tono llevado hasta ahora, esta preponderancia de los derechos humanos también tendría que romper con las limitaciones espaciales clásicas y adaptarse a la realidad global: tendría que constituirse como parte de una verdadera esfera pública global. El nuevo paradigma radicaría en que cada unidad política del plano global procurase el imperio de estos derechos en la esfera transnacional. Ningún proyecto de Estado puede estar ni mínimamente completo si limita la procuración de los derechos humanos tan solo a sus ciudadanos y obtiene beneficios, aunque sea de manera derivada, de la vulneración que de estos derechos sufren los ciudadanos de otros Estados.

Ibid., p. 33.

44 David Held, Democracy and the Global Order: From the Modern State to Cosmopolitan Governance, Stanford University Press, Stanford, 1995, p. 281. 
El cómo de una globalización política también estaría limitado a aspirar a un esquema de mínimos. En este sentido, probablemente el mejor camino sea el de buscar potenciar la cooperación entre las naciones existentes sin pretender la creación de un organismo supranacional que sea capaz de someter a los Estados y arregle todos los problemas.

La solución no consiste en construir una esfera pública supranacional, sino en transnacionalizar las esferas públicas nacionales existentes. De esta forma las últimas serán más responsivas entre ellas sin necesidad de cambios drásticos en la infraestructura actual. Al mismo tiempo, las barreras de las esferas públicas nacionales podrían convertirse en ventanas para un entendimiento mutuo ${ }^{45}$.

Se trata pues de buscar algo similar a lo que Martin Albrow llama el Estado global, uno que se constituya a partir de una red policéntrica de prácticas a nivel global ${ }^{46}$, un Estado que se siga los lineamientos expuestos por Ulrich Beck (1944-2015) cuando habla de "Estados transnacionales", es decir, de unos Estados mucho más dispuestos a la cooperación y que hagan de la globalización un proyecto político ${ }^{47}$. Un escenario así no requiere de un sometimiento ideológico "occidentalizador", ni de la resolución de antagonismos milenarios, sino de un entendimiento en común de que hay problemas globales y déficits de poder que solo pueden ser cubiertos de manera conjunta. No hay una pretensión "occidentalizadora" en ese sentido, pero sí parece indispensable la participación de las principales naciones que integran tal polo ideológico, pues a pesar de que cada vez son más las fuerzas políticas que preferirían enfrentar la globalización haciendo que sus naciones adoptasen la forma de comunidades cerradas (Global Gemeinschaft), siguen siendo estas naciones "occidentales" las más propicias a mantenerse como sociedades abiertas (Global Geisellschaft) ${ }^{48}$. Sin embargo, para que sea posible alinear la voluntad de tales naciones, o de sus ciudadanos, es necesario dotar de entera realidad a los ideales ilustrados sobre los que se formaron esos proyectos de Estado democrático constitucional. Estos ideales no pueden seguir encontrándose meramente en el papel como cartas de buenas intenciones o como parte de una protocolaria moral política ejercida solo de dientes para afuera y frente a las cámaras. Cierto nivel de congruencia en la política de estas naciones es necesario para que los ciudadanos que se encuentran bajo su cobijo comulguen, aunque sea también en un esquema de mínimos, con estos ideales. Solo entonces parece que será posible continuar con la edificación de los numerosos aspectos procedimentales que aún se encuentran pendientes en los ámbitos local y global de este proceso de redefinición de lo político.

\section{Referencias bibliográficas}

Albrow, Martin, The Global Age: State and Society Beyond Modernity, Polity Press, Cambridge, 1996.

\footnotetext{
5 Habermas, Europe: The Faltering Project, p. 183.

46 Albrow, The Global Age, pp. 178-180.

47 Ulrich Beck, ¿Qué es la globalización? Falacias del globalismo, respuestas a la globalización, Paidós, Barcelona, 2008, pp. 206-216.

48 Robertson, Globalization, pp. 78-79.
} 
Appadurai, Arjun, Modernity at Large: Cultural Dimensions of Globalization, University of Minnesota, Minneapolis, 2009.

Beck, Ulrich, La sociedad del riesgo, Paidós, Barcelona, 1998.

- ¿Qué es la globalización? Falacias del globalismo, respuestas a la globalización, Paidós, Barcelona, 2008.

—, World at Risk, Polity Press, Cambridge, 2009.

Beckstead, Nick, y Ord, Toby, "Managing Existential Risk from Emerging Technologies", en Mark Peplow (ed.), Annual Report of the Government Chief Scientific Advisor 2014. Innovation: Managing Risk, Not Avoiding It, Government Office for Science, London, 2014, pp. 115-120.

Bostrom, Nick, "Existential Risk Prevention as Global”: Global Policy, vol. 4, n. ${ }^{\circ}$ (2013), pp. 15-31.

Casey, Michael, "Can this Woman Convince the World to Act on Climate Change?": CBS (2-10-2015). Disponible en: https://goo.gl/DK4Rvt (6-7-2017).

Cotton-Barratt, Owen, et. al., Global Catastrophic Risks 2016, The Global Challenges Foundation, Global Priorities Proyect, 2016. Disponible en: https://goo.gl/8w5ZYs (6-7-2017).

FitzRoy, Felix R., y Papyrakis, Elissaios, An Introduction to Climate Change Economics and Policy, Routledge, London, 2016.

Fraser, Nancy, "Transnationalizing the Public Sphere: On the Legitimacy and Efficacy of Public Opinion in a Post-Westphalian World", en Nancy Fraser et al., Transnationalizing the Public Sphere, Polity Press, Cambridge, 2014.

Habermas, Jürgen, Europe: The Faltering Project, Polity Press, Cambridge, 2009.

Held, David, Democracy and the Global Order: From the Modern State to Cosmopolitan Governance, Stanford University Press, Stanford, 1995.

Hickman, Leo, “Timeline: How BECCS Became Climate Change's 'Saviour' Technology”: Carbon Brief (13-4-2016). Disponible en: https://goo.gl/XwkgfU (6-7-2017).

IPCC, Climate Change 2014: Impacts, Adaptation, and Vulnerability. Part A: Global and Sectoral Aspects. Contribution of Working Group II to the Fifth Assessment Report of the Intergovernmental Panel on Climate Change, Cambridge University Press, Cambridge. Disponible en: https://goo.gl/xdWxzP (6-7-2018).

-, Climate Change 2014: Mitigation of Climate Change. Contribution of Working Group III to the Fifth Assessment Report of the Intergovernmental Panel on Climate Change, Cambridge University Press, Cambridge, 2014. Disponible en: https://goo.gl/ZKho2K (6-7-2017).

Krever, Mick, "U.N. Climate Chief: Countries Acting for Economy, Not Planet”: CNN (1-122015). Disponible en: https://goo.gl/5KLw8k (6-7-2017);

Kugelman, Michael "Kashmir Conflict: Do Nuclear Weapons Prevent All-out War?": CNN (30-9-2016). Disponible en https://goo.gl/jZU7NG (6-7-2017).

Mecklin, John (ed.), "It is Still 3 Minutes to Midnight, 2016 Doomsday Clock Statement": Bulletin of the Atomic Scientists (2016). Disponible en: https://goo.gl/fTVS64 (7-6-2017).

—, "It is Two and a Half Minutes to Midnight, 2017 Doomsday Clock Statement": Bulletin of the Atomic Scientists (2017). Disponible en: https://goo.gl/o3XFkz (28-9-2017).

Olivier, Jos G. J., et al., Trends in Global $\mathrm{CO}_{2}$ Emissions: 2015 Report, PBL Netherlands Environmental Assessment Agency, The Hague, 2015. Disponible en: https://goo.gl/pMJUV2 (6-7-2017).

Paltsev, Sergey, “The Complicated Geopolitics of Renewable Energy": Bulletin of the Atomic Scientists, vol. 72, n. ${ }^{\circ} 6$ (2016), pp. 390-395. Disponible en: https://doi.org/10.1080/00963402.2016.1240476 (6-7-2017). 
Reilly et al., Energy \& Climate Outlook. Perspectives From 2015, Massachusetts Institute of Technology, Cambridge, 2015. Disponible en: https://goo.gl/6JREiQ (6-7-2017).

Rodrik, Dani, The Globalization Paradox, Norton, New York, 2011.

Robertson, Roland, Globalization: Social Theory and Global Culture, SAGE Publications, London, 1992.

Rosanvallon, Pierre, Por una historia conceptual de lo político, Fondo de Cultura Económica, Buenos Aires, 2003.

-, La contrademocracia: La política en la era de la desconfianza, Manantial, Buenos Aires, 2007.

Rosenau, James N., Turbulence in World Politics. A Theory of Change and Politics, Princeton University Press, Princeton, 1990.

-, The Study of the World Politics, Volume 2: Globalization and Governance, Routledge, London, 2006.

Shukman, David, "Hawking: Humans at Risk of Lethal 'Own Goal"”: BBC (19-1-2016). Disponible en: https://goo.gl/XfXt5J (6-7-2017).

Stiglitz, Joseph E., "Overcoming the Copenhagen Failure with Flexible Commitments": Economics of Energy and Environmental Policy, vol. 4, n. ${ }^{\circ} 2$ (2015), pp. 29-36.

UNFCCC, Climate Action Now: Summary for Policymakers 2017, United Nations Climate Change Secretariat, Bonn, 2017. Disponible en: https://goo.gl/LfYRDw (6-7-2017).

Vallespín, Fernando, El futuro de la política, Taurus, Madrid, 2000.

Wallerstein, Immanuel, The politics of the World-economy, Cambridge University Press, Cambridge, 1984. 